\title{
A Trap Motion in Validating Muscle Activity Prediction from Musculoskeletal Model using EMG
}

\author{
Wibawa, A. $\mathrm{D}^{1}$, Verdonschot, $\mathrm{N}^{5,6}$, Halbertsma, J. P. $\mathrm{K}^{2}$, Burgerhof, J. G. $\mathrm{M}^{4}$, \\ Diercks, R. $\mathrm{L}^{3}$ and Verkerke, G. $\mathrm{J}^{2,6}$ \\ ${ }^{1}$ Department of Multimedia and Network Engineering, Institut Teknologi Sepuluh \\ Nopember, Surabaya, Indonesia \\ ${ }^{2}$ Rehabilitation Medicine, ${ }^{3}$ Orthopaedics, ${ }^{4}$ Epidemiology, University of \\ Groningen, University Medical Center Groningen, the Netherlands \\ ${ }^{5}$ Orthopaedics Research Laboratory, Radboud University Medical Centre, \\ Nijmegen, the Netherlands \\ ${ }^{6}$ Department of Biomechanical Engineering, University of Twente, Enschede, the \\ Netherlands \\ Telephone: +62 31592 2936; email: adhiosa@te.its.ac.id
}

\begin{abstract}
Musculoskeletal modeling nowadays is becoming the most common tool for studying and analyzing human motion. Besides its potential in predicting muscle activity and muscle force during active motion, musculoskeletal modeling can also calculate many important kinetic data that are difficult to measure in vivo, such as joint force or ligament force. This paper will validate muscle activity predicted by the model during a static motion like knee flexion motion (squat motion). In this experiment, knee flexion motion was performed by 5 healthy subjects and modeled by using Gait Lower Extremity model from AnyBody Modeling System (AMS). Eight lower limb muscle activity prediction from the model will be validated by 8 EMG electrodes that measured the same muscles during squat motion. Muscle activity pattern and the position of onset would be used as a key factor in this validation study. Pearson correlation coefficient will be used to compare the pattern of both graphs. Knee joint force prediction from the model will also be compared with the literature studies. The result showed that 3 muscles showed high correlation coefficient, while the other 4 muscles showed slightly medium and one showed low correlation. Time delay of muscle activation between the model and EMG was recorded from Vastus Medialis muscle (18.38 ms) and Vastus Lateralis $(22.8 \mathrm{~ms})$, with muscle activation from the model was late compared to EMG. In conclusion, this statistical study has shown some detail differences between EMG and muscle activity prediction from the model. Knee flexion motion can be used as a trap motion when validating muscle activity of a musculoskeletal model, because the model will activate muscle activity based on motion data of markers, while in knee-flexed position, there was no marker's movement, but the EMG was highly active due to the posture of the subjects in maintaining the kneeflexed position. However, the knee compressive force prediction from the model has showed positive confirmation from the literatures.
\end{abstract}

Keywords: Knee flexion motion, musculoskeletal modeling, Inverse dynamics analysis, muscle activity validation, EMG

\section{Introduction}

Studying human biomechanics especially during active movement is still challenging nowadays. This is explained by the fact that muscle activation during active motion is controlled by our central nervous system (CNS) in which until now, the exact mechanism 
remains unclear [6]. In Addition, some important data such as knee joint forces and joint moments that determined significantly the cause of most knee injuries during movement $[14,21]$ are difficult to obtain. Due to that condition, an alternative way in accessing those important data is by using a prediction tools such as a musculoskeletal modeling application [3]. From our previous study, we validated and analyzed three motion (normal walking, one legged forward hopping and side jumping). During those studies, muscle activity prediction from the model was validated by EMG using three variables of a graph; number of onset, offset and peaks. The result of validation during normal walking was low, however, during more prescribed movement like forward hopping and side jumping, the validation result was better [27]. Some conclusive points were obtained from those studies, such as predicted muscle activity by AMS was always late compared to EMG and EMG is always longer in duration compared to the predicted muscle activity by AMS. However, detail calculation on how much the model late was not yet done.

From those experiences, we found that type of movement was taking a significant role in determining the level of agreement between the model with EMG, besides number of assumptions and simplifications in the modeling process. By referring to that result a simplest motion like knee flexion motion may show a clearer result of agreement between modeling and EMG. Knee flexion motion is the basic human motion from human's early age. There were many studies that explored the biomechanical aspects during this motion [8] and the potential of this motion in describing the human mechanics especially for patients post Total Knee Arthroplasty (TKA) surgery [7] or patients with Osteoarthritis (OA) or rheumatoid arthritis [13]. Other modeling studies in approximating knee joint forces or muscle forces during knee flexion were also done in the past [9], a robotic simulation study by using cadavers in approximating knee kinematics and kinetics was also done previously by [12]. However, analyzing and comparing the predicted muscle activity by using Gait Lower Extremity model from AMS with EMG and related the analysis with knee joint forces prediction is still a new insight in the field of musculoskeletal model validation.

The main goal of this study is exploring the level of agreement of muscle activity prediction from the model with EMG during knee flexion motion by using Pearson correlation coefficient calculation. We hipothesized that knee flexion motion can be used as a trap motion in validating muscle activity prediction from the model since the prediction of the model is based on marker's movement data and ground reaction force. Time delay between onset timing of EMG with the model will be calculated. The second goal is analyzing knee joint forces and moments resulted from the models, and compare them with the result from the previous studies. At conclusion, this paper will analyze to which circumstance AMS predict better or less in term of muscle activity compared to our previous studies.

\section{Method}

\subsection{Subjects}

This study was involving 5 healthy subjects performing knee flexion motion. Subject's age were above 18 years, and below 50. Subject who has pain in the knee or other lower limb parts that could cause abnormal knee flexion is excluded, including having lower limb trauma, neurological or metabolic disorders that can effect on lower limb functioning (diagnosed by a sports physician). The characteristics of subjects ( 2 males and 3 females) were: mean age of $27.8 \pm 5$ years, mean body weight $63.6 \pm 5.6 \mathrm{~kg}$. The study was approved by the Medical Ethical Committee of the University Medical Center Groningen (UMCG). Each subject signed an inform consent before performing the experiment.

This experiment was performed in a Gait Laboratory, Centre for Rehabilitation Medicine, UMCG, Groningen, The Netherlands. A $9.0 \mathrm{~m}$ long walkway was prepared. 
Two force plates (AMTI) were embedded on the floor to measure the ground reaction force (GRF) with a sampling frequency of $1000 \mathrm{~Hz}$. Two cameras (Basler A602 FC) in fixed positions were used to record the motion with sampling frequency of $50 \mathrm{~Hz}$. Vicon system was used to record, synchronize and analyse the motion. Sixteen reflective markers were attached to bony landmarks on both lower limbs so that the eight infrared cameras could track and record the trajectories of the markers during motion. The rule for placing the markers was based on the study of Hayes and Davis [4].

Non-invasive EMG Zerowire electrodes (Aurion SRL) were used for subject's comfort to record the muscle activity of the right lower limb. The skin was prepared and cleaned before attaching the EMG electrodes on the muscles: Rectus Femoris (RF), Vastus Medialis (VM), Vastus Lateralis (VM), Semitendinosus (ST), Bicep Femoris (BF), Gastrocnemius Medialis (GM), Gastrocnemius Lateralis (GL) and Tibialis Anterior (TA). Seniam standard placement was used as a guidance for EMG electrodes placement [15].

\subsection{Protocol and Modeling the Motion}

The subjects were asked to stand on the plate form for the first position, and wait for a sign before performing a flexion. The angle of knee flexion is approximately $45^{\circ}$. When a sign was given, subjects performed knee flexion and maintain that position for about 5 seconds then rise up again, back to standing position as previously. This performance was done two or three times in each trial. Figure 1 showed the steps during knee flexion motion and figure 2 shows the model during knee flexion. A C3D file which was obtained during experiment, consists of marker trajectories and ground reaction force data that will be used to model the motion.

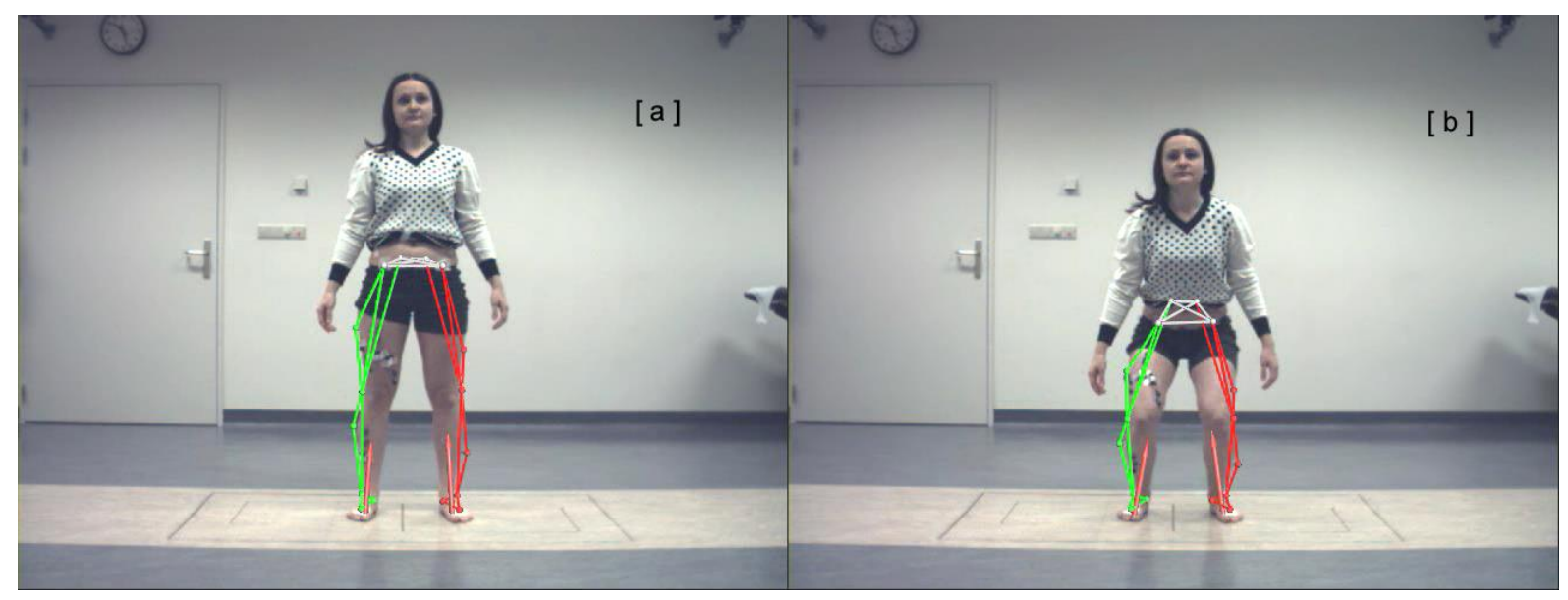

Figure 1. (a) Standing Position Before Knee Flexion; (b) Subject Maintains the Knee-flexed Position; 


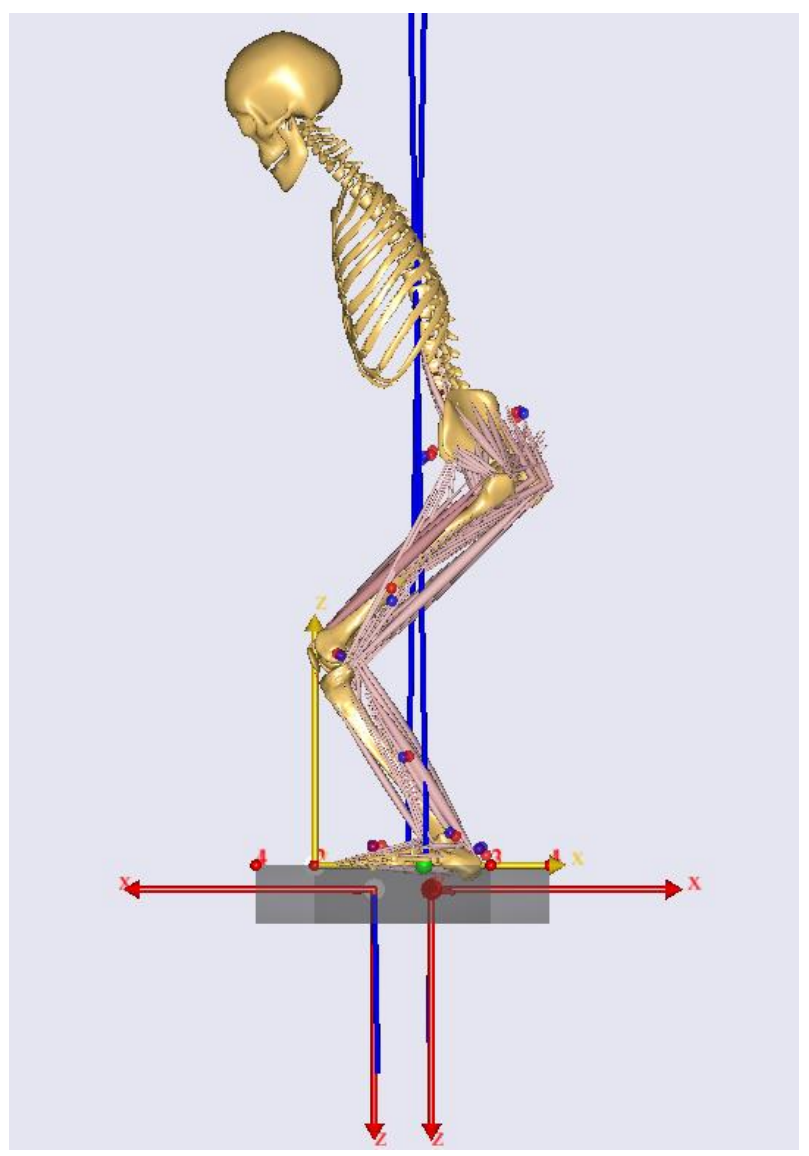

Figure 2. Musculoskeletal Model of Knee Flexion

In this study, Gait Lower Extremity Model - GLEM (AMMR.1.3.1) from AnyBody Modeling System version 5.0 was used to model the knee flexion motion. GLEM consists of lower limb bones, including pelvis, with all muscles and tendons are attached, but without upper body [27]. Anthropometric data such as body weight, body height, pelvis, thigh, shanks and foot length were imported from the subject measurement. The default scaling algorithm which is based on mass-fat scaling algorithm was applied in the model [1]. In GLEM, the knee was modeled as a hinge between femur and tibia bone. This type of the knee enables only movement in the sagittal plane. AMS modeling uses inverse dynamic algorithms to predict muscle activity and all knee joint forces in 3 directions (anterior-posterior: AP, medial-lateral: ML, proximal-distal: compressive force) and 2 knee joint moments (axial moment and varus-valgus moment). The orientation of the 3 knee joint forces and 2 knee joint moments are defined from femur coordinate system as described in fig 3 . 


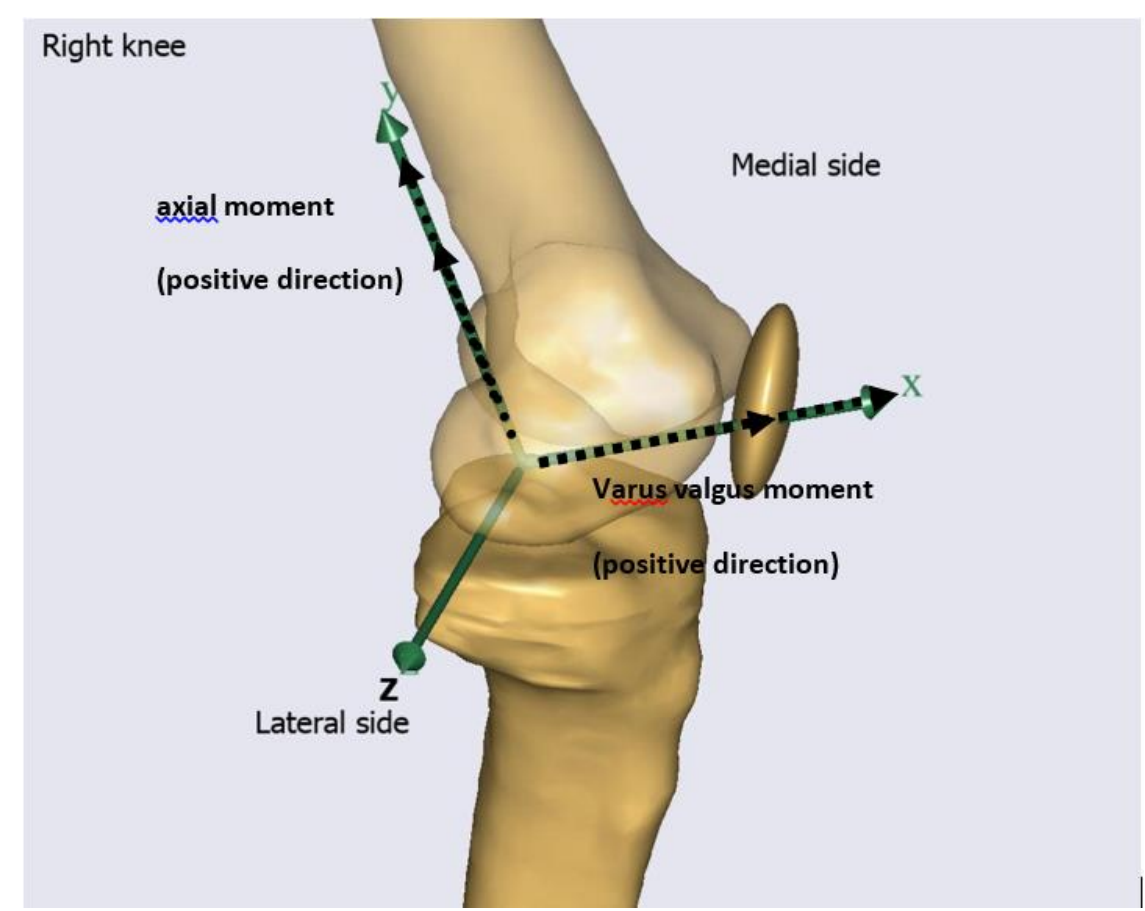

\section{Figure 3. Femur Coordinate System for Determining the Knee Joint Forces Direction}

C3D file resulted from lab measurement contains raw EMG data. During simulation, AMS is also capable of doing rectifying and filtering the raw EMG data into smoothed EMG. All parameters of filtering EMG is editable in AMS script files. In this study, AMS filtered the raw EMG using Butterworth band pass filter [5] with a frequency range of 30$200 \mathrm{~Hz}$ and $6 \mathrm{~Hz}$ low pass filter [17], so that when inverse dynamic analysis is finished, then the measured EMG is also ready for comparison.

\subsection{Data Analysis}

From 5 subjects who performed 3 trials of knee flexion motion, there should be 15 knee flexion models for analysis, but due to some marker errors during experiment, there were only 13 knee flexion models. The analysis of knee joint forces and moments prediction was done in each plane (see figure 3). Each plane contains two values, negative and positive. This value is actually representing the direction of the predicted knee joint force relative to femur coordinate system whether they show distally or proximally (see fig 3 above), or anteriorly/posteriorly. For example, in figure 3, knee joint force is pointing out at positive $\mathrm{X}$ direction, this is assumed by the model as the anterior direction, vice versa condition for posterior direction. The predicted knee joint moments are also being assumed similarly in the model. For example, the knee axial moment, when the direction of this moment is positive, this means that the moment is rotating laterally. Opposite condition happened for negative knee axial moment. For the knee varus-valgus moment, its original name generated by AMS is actually knee lateral moment, meaning that all positive value of this moment will be define as moment that pointing out at lateral direction (varus) and vice versa for valgus moment.

\subsection{EMG Comparison}

Activation level of EMG is specific for each subjects. This level depends greatly on subject's anatomical condition ${ }^{5}$, therefore it is important to process the EMG graph, especially its baseline threshold, into a more reproducible way. In this study, before the 
EMG was being compared with AMS by using Pearson correlation coefficient calculation, the data was cut from the minimum value (the lowest point of the graph) until the maximum value of the graph. So the baseline was determined from the lowest EMG data for each muscle [17].

For the predicted muscle activity from the model, baseline threshold was determined by value $10^{-7}$, meaning that all values below $10^{-7}$ will be zeroed. This was because the data of predicted muscle activity from AMS never reach a zero level, so we need this threshold to define the zero level. When both data (EMG and predicted muscle activity) were ready then Pearson correlation coefficient calculation was performed to find their level of agreement in term of graph pattern. Onset time point of EMG will be used as reference for calculating the delay of muscle activity predicted by the model.

\section{Result}

Typical EMG and predicted muscle activity by AMS of 8 muscles during knee flexion motion was described in figure 4-5.

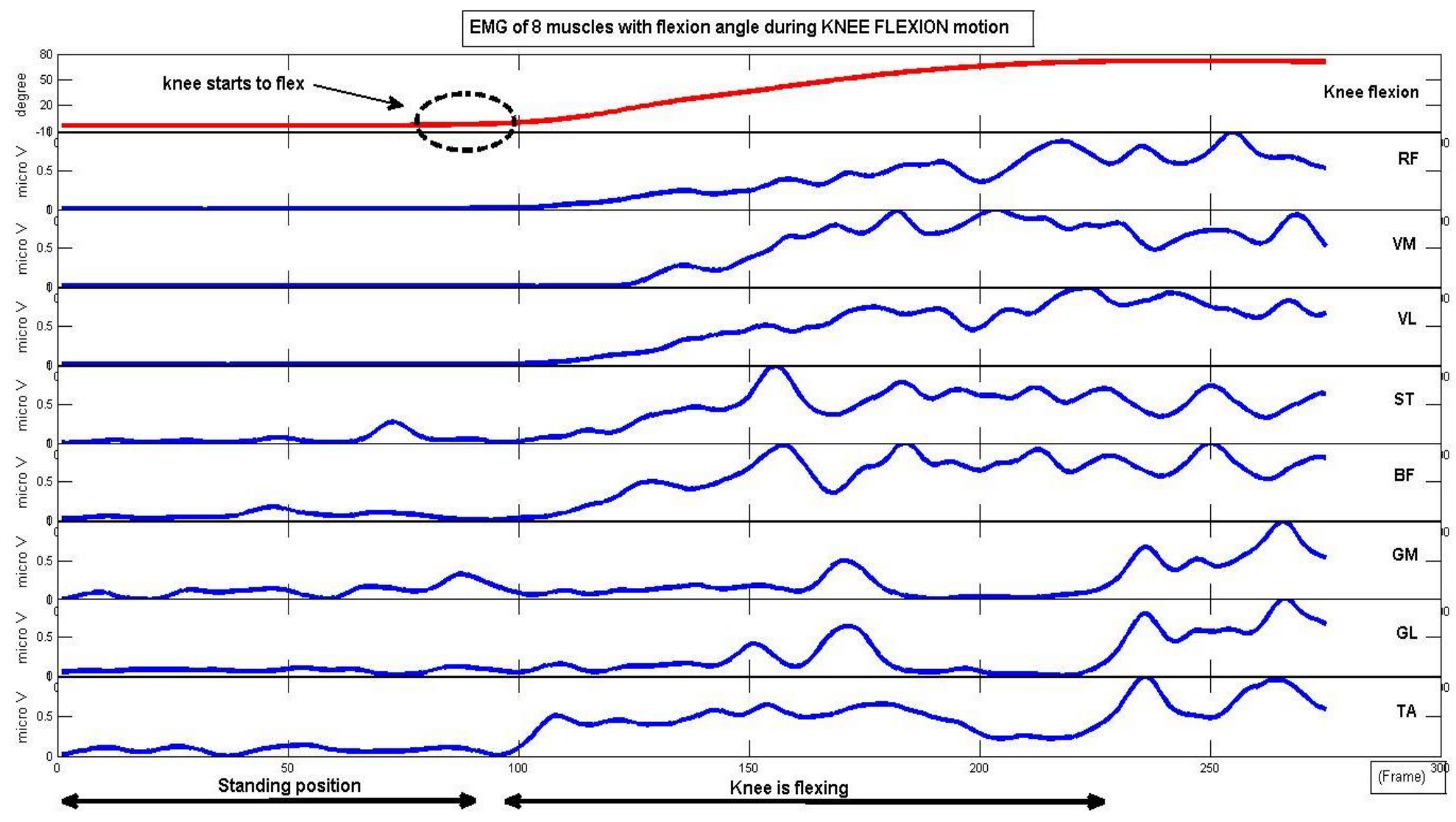

Figure 4. Typical Muscle Activity from EMG During Knee Flexion (Red Graph is Knee Flexion Angle During Knee Flexion Motion) *Frame is Equal to Milisecond 


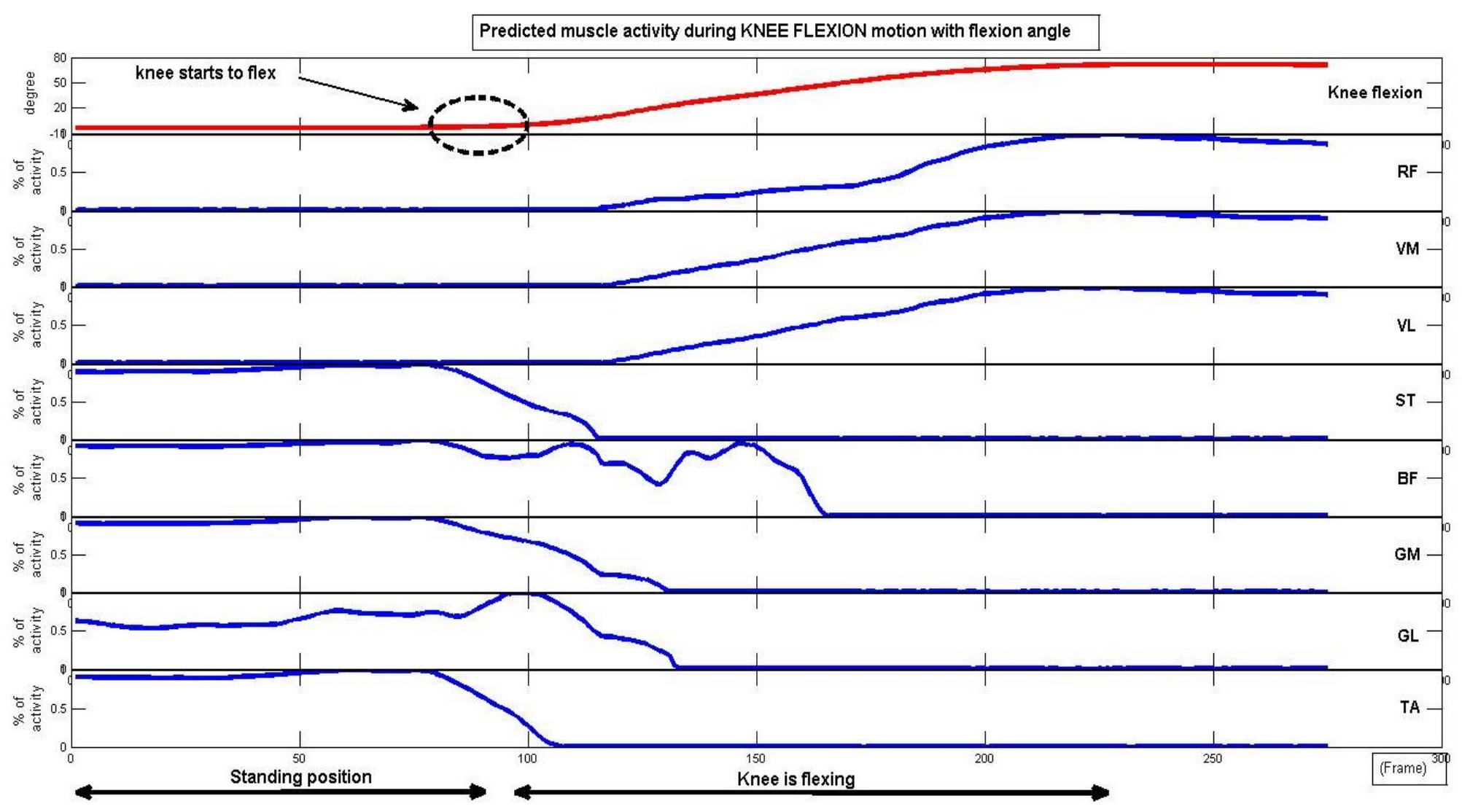

Figure 5. Typical Predicted Muscle Activity from AMS During Knee Flexion (Red Graph is Knee Flexion Angle During Knee Flexion Motion) *Frame is Equal to Milisecond

Knee joint forces and moments prediction during knee flexion motion was showed in figure 6. This figure was showing the mean of maximum knee joint forces or moments from all 13 models.

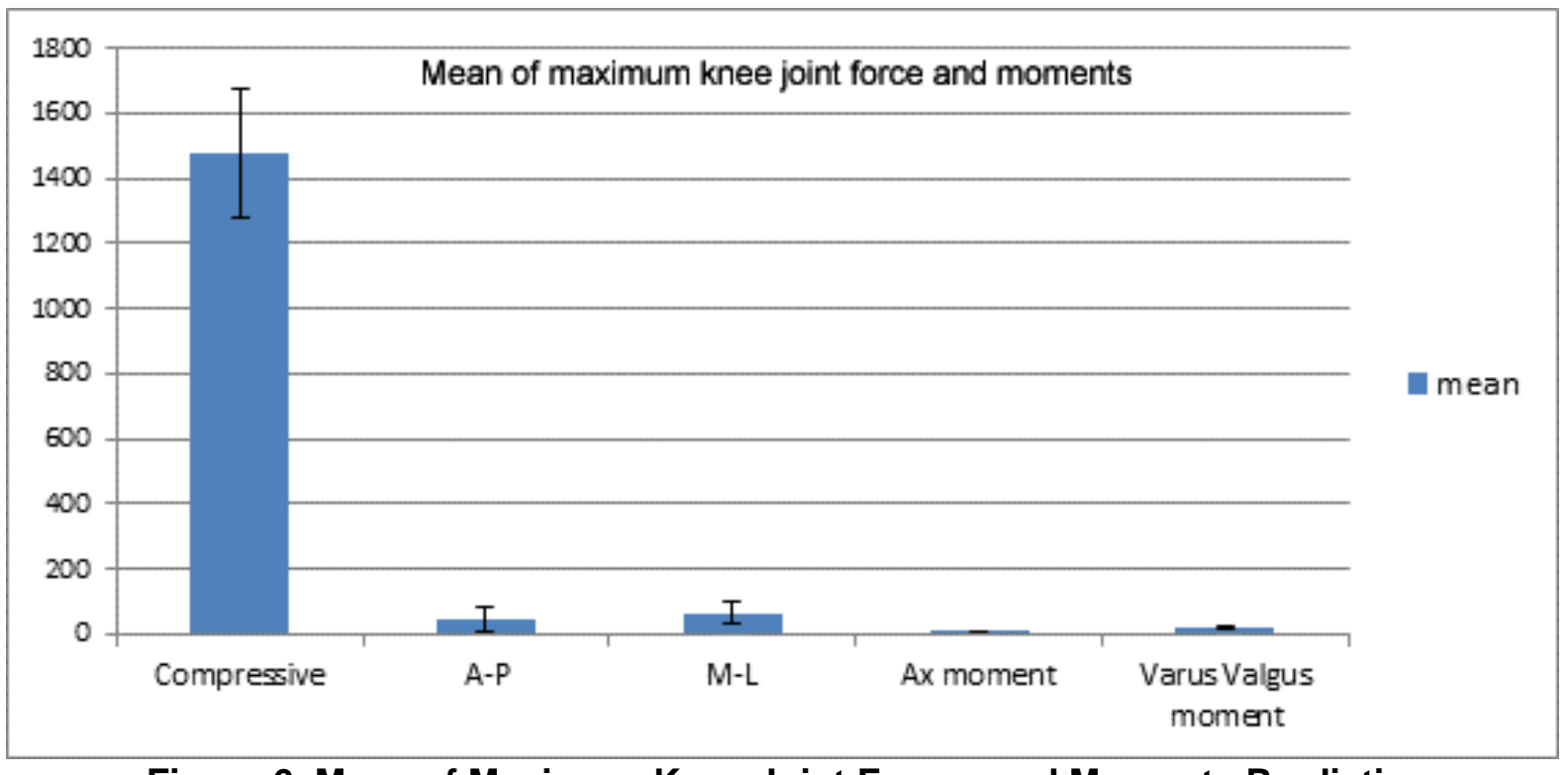

Figure 6. Mean of Maximum Knee Joint Forces and Moments Prediction During Knee Flexion Motion ${ }^{\star}$ Compressive is Knee Compressive force (CF) 
Knee compressive force within model variation was presented in relation with body weight (BW) (see figure 7 below).

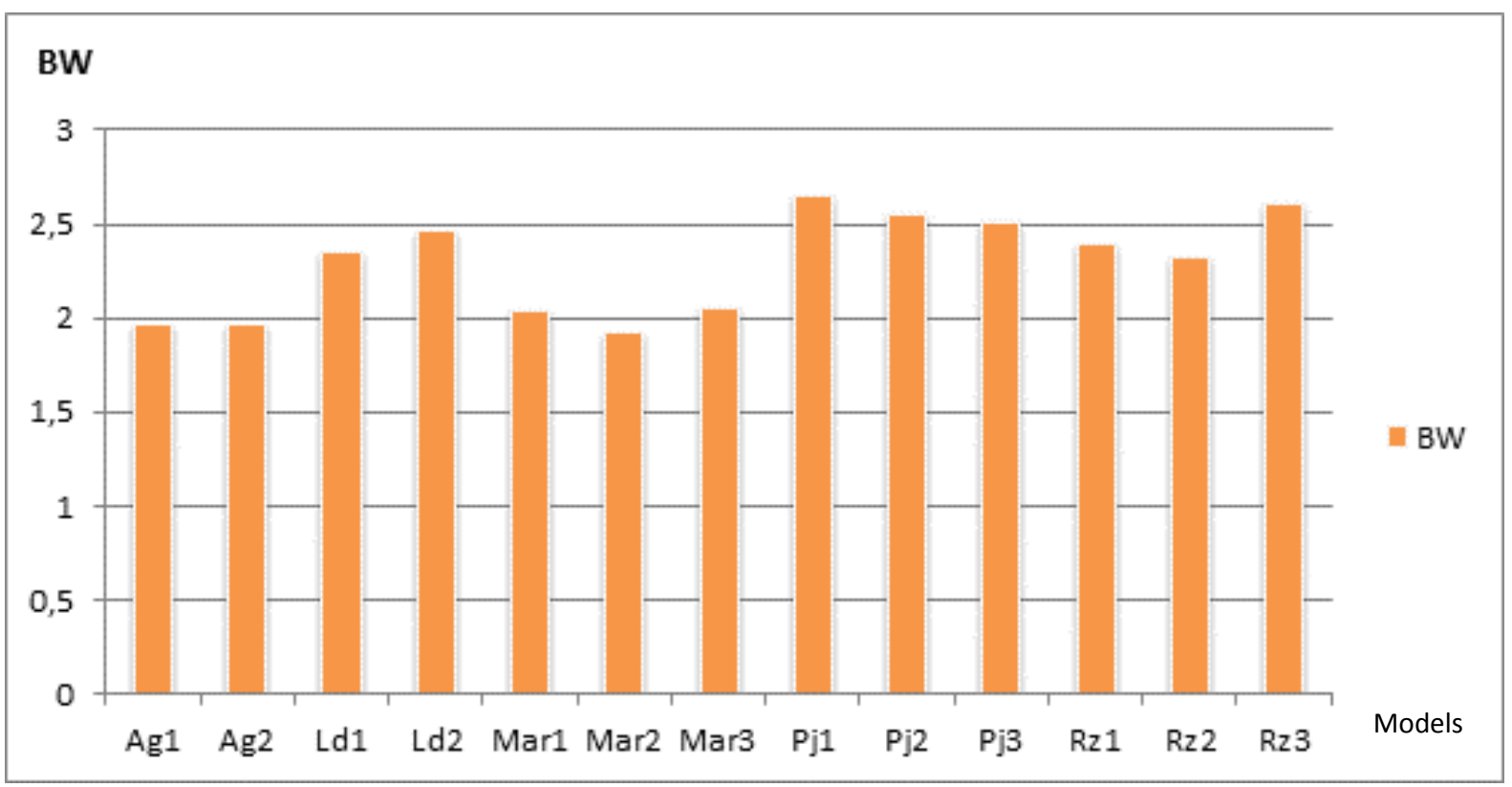

Figure 7. Model Variation in Knee Compressive Force During Knee Flexion Motion

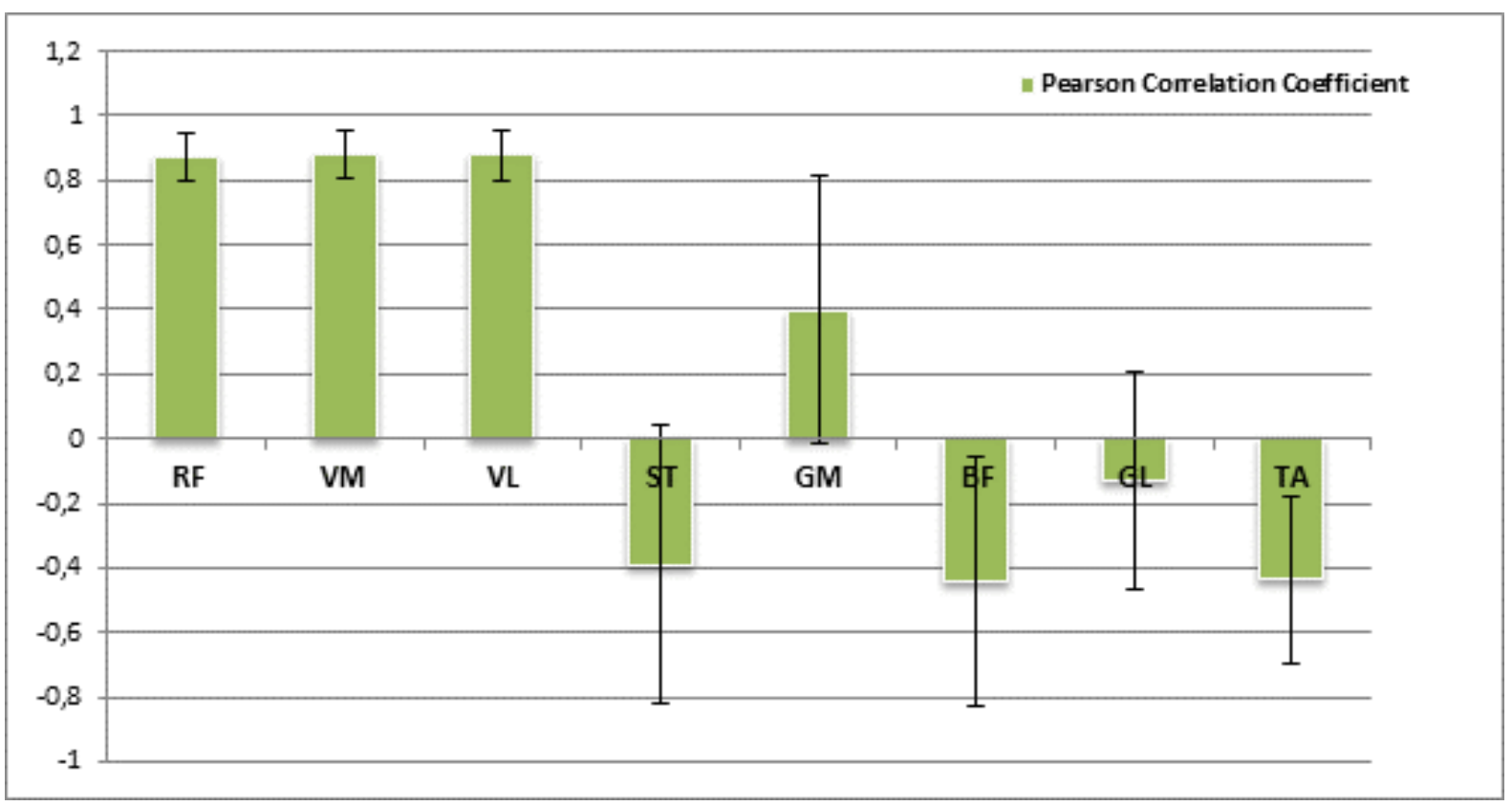

Figure 8. Pearson Correlation coefficient between Predicted Muscle Activities by The Model with EMG from all Eight Muscles During Knee Flexion Motion

From our previous study, we found that EMG is always longer and initiated earlier before AMS during normal walking, forward hopping and side jumping activities [27]. In this knee flexion modeling, some muscles like VM and VL can show a clear onset timing from standing position, until subject was in position maintaining knee-flexed position. So these two muscles were used to calculate the time delay of muscle activation between 
EMG and AMS. By calculating the mean of time delay happened from all models in VM and VL muscle, we found that for VM muscles, the mean of time delay was calculated as $18.38 \mathrm{~ms}$, and for VL muscle was $22.8 \mathrm{~ms}$.

\section{Discussion and Conclusion}

This discussion part was divided into three stages of analysis, during standing position, transient condition when the knee is flexing, and when the knee maintains the flexing condition. Stage 1, during standing position, from figure 4-5 we can see how the model predicted muscle activity, comparing to EMG activity. Some differences happened at this stage. In EMG for example, there was only small activities from muscle ST, BF, GM, GL and TA during standing. Compared to AMS, contrary condition happened in the model due to the significant activities of the same muscles. Continuing the observation into stage 2, during flexing motion. Considering the timing when the knee was about to flex, in EMG level we saw ST, BF and TA muscles were increasing the activity gently, then being continued by RF, VM and VL at some frames later. Similar condition happened in predicted muscle activity especially on muscles: RF, VM and VL (see figure 5). However, for muscle ST and BF, the activity was even opposing EMG. On the stage 3, when the knee was keeping the flexing position, EMG of all muscles remain active (ST, BF, RF, VM, VL and TA). Additional activities were showed by GM and GL muscles in holding the knee position and keeping the balance of the upper body from falling. In contrast, different conditions were shown by the model, while during the same timing, ST, BF, GL, GM and TA were inactive. There were only three muscles that showed similar pattern as EMG in this time frame, they were RF, VM and VL (see figure 4-5). This result was projected by figure 8 , where the Pearson correlation coefficient for muscles: RF, VL and VM were higher than the other 5 muscles.

More over during stage 3, when the knee was keeping the flexed position, the marker's movement data was absent, but the lower limb muscles were tensioning because they must hold the whole upper body from falling. We think that this position can cause error in the model's prediction especially in calculating muscle activity, because in the calculation process, model needs the marker's movement data to calculate the velocity and acceleration of a body segment. By combining them with the GRF data, model then can predict the force that is needed to move a body segment, including muscle activity. This knee flexion experiment, to our opinion, is a trap motion for the model in predicting muscle activity. We speculated that this was mostly caused by the absence of cocontraction muscles function in the model. As we can see in flexing condition, ST and BF not only handle the impact of upper body part (including the pelvis) [20] but also functioning as knee flexor. Setting this double function to some muscles at the same time is challenging in term of modeling especially in this modeling version (AMMR 1.3.1). These findings certainly provide significant difference between muscle activity prediction by the model with the EMG.

In addition, there are also some other factors that may cause the low level of agreement between the model's prediction and EMG such as uncertainty of inverse dynamics analysis like what has been studied by [25]. This uncertainty can be caused by several aspects like inaccuracy in segmental parameter (including mass, moment of inertia and center of mass) [11], inaccuracy in marker's data such as noise in surface marker [24] or ground reaction force [18] and inaccuracy in determining (predicting) the location of joint center [2,19]. All of those factors for now is still a challenging tasks in term of modeling.

In this study, a time delay was recorded between EMG and AMS. In this finding, we used only two muscles (VM and VL) to calculate time delay because only from these two muscles, the activation time point was clearly shown (see figure 4-5). This information is useful for future validation study or for a clinical use that the muscle activity from the model should be calculated about 20 ms later compare to EMG. The existance of delay is 
confirmed well by the previous review study done by [28]. In that previous study, some adjusment strategy need to be performed when comparing EMG and muscle activity prediction from the model.

Considering the knee compressive force (CF) analysis, figure 6 showed clearly that knee $\mathrm{CF}$ was the main knee joint forces working in the knee during knee flexion motion, this is because when we compare knee CF to the other knee joint forces such as knee anterior-posterior force or knee medial-lateral force, knee CF was way higher. This result was different compared to our previous study during forward hopping and side jumping modeling. In those studies, knee anterior-posterior force (knee A-P force) was showing at about 0.5 of the predicted knee CF. When comparing to this study, knee A-P force was below $10 \%$ of knee CF prediction. Mean of knee CF in relation with BW that was calculated from figure 7, was reported 2.29 BW. Compared to other studies [16] this result was similar to some previous studies that investigated the walking compressive force as reported by $[10,22,25,26]$. This is a positive point from this modeling software to the science because then we can use it analysis tool for many clinical applications such as early detection in the knee ligament disorder or rehabilitation.

Despite low result in agreement using EMG and the fact of time delay, however, the knee compressive force prediction by model reach similar result as predicted by other studies. We conclude that AMS model predicts well muscle activity in more dynamics circumstance or more prescribed movement (referring to our previous validation studies during forward hopping and side jumping) rather than in a statics movement like knee flexion motion. The AMS model has a great potential for a clinical use when considering the knee compressive force analysis. Some additional features like adapting cocontraction muscle function and later timing of muscle activation during modeling have been proposed from this paper so that more precise prediction can be achieved in the future. A validation study that involves more participants from different race may yield a better knowledge in comparing muscle activity predicted by the model with EMG in the future.

\section{Conflict of Interest}

There is no conflict of interest in sponsoring this study.

\section{Acknowledgement}

This study was sponsored by the Directorate General of Higher Education (DIKTI), Ministry of National Education, Indonesia and the University of Groningen, the Netherlands.

\section{References}

[1] M. S. Andersen, M. Damsgaard, B. Macwilliams and J. A. Rasmussen, "A computationally efficient optimisation-based method for parameter identification of kinematically determinate and overdeterminate biomechanical systems", Computer Methods in Biomechanics and Biomedical Engineering, vol. 13, no. 2, (2010), pp. 171-83.

[2] A. L. Bell, D. R. Pedersen and R. A. Brand, "A comparison of the accuracy of several hip center location prediction methods", Journal Biomech, vol. 23, no. 6, (1990), pp. 617-21.

[3] M. Damsgaard, J. Rasmussen, S. T. Christensen, E. Surma and M. D. Zee, "Analysis of musculoskeletal systems in the AMS Modeling System", Simulation Modelling Practice and Theory, vol. 14, (2006), pp. $1100-1111$

[4] R. B. Davis III, S. Ounpuu, D. Tyburski and J. R. Gage, "A gait data collection and reduction technique. Human Movement Sciences, vol. 10, (1991), pp. 575-587.

[5] C. J. De Luca, L. D. Gilmore, M. Kuznetsov and S. H. Roy, "Filtering the surface EMG signal: Movement artifact and baseline noise contamination", Journal of Biomechanics, vol. 43, (2010), pp. 1573-1579

[6] T. G. Deliagina, P. V. Zelenin, I. N. Beloozerova and G. N. Orlovsky, "Nervous mechanisms controlling body posture", Physiology \& Behavior, vol. 92, (2007), pp. 148-154. 
[7] D. A. Dennis, R. D. Heekin, C. R. Clark, J. A. Murphy, T. L. O'Dell and K. A. Dwyer, "Effect of Implant Design on Knee Flexion", The Journal of Arthroplasty, vol. 28, (2013), pp. 429-438.

[8] A. Erdemir, S. McLean, W. Herzog and A. J. Van den Bogert, "Model-based estimation of muscle forces exerted during movements", Clinical Biomechanics, vol. 22, (2007), pp. 131-54.

[9] E. Forster, U. Simon, P. Augat and L. Claes, "Extension of a state-ofthe-art optimization criterion to predict co-contraction", Journal Biomech., vol. 37, (2004), pp. 577-581.

[10] B. J. Fregly and F. E. Zajac, "A state-space analysis of mechanical energy generation, absorption, and transfer during pedaling", Journal of Biomechanics, vol. 29, no. 1, (1986), pp. 81-90.

[11] K. J. Ganley and C. M. Powers, "Determination of lower extremity anthropometric parameters using dual energy X-ray absorptiometry: the influence on net joint moments during gait", Clin Biomech, vol. 19, no. 1, (2004), pp. 50-60.

[12] G. Li, S. Zayontz, L. E. DeFrate, E. Most, J. F. Suggs and H. E. Rubash, "Kinematics of the knee at high flexion angles: an in vitro investigation", Journal of Orthopaedic Research, vol. 22, (2004), pp. 90-95

[13] K. Harato, T. Nagura, H. Matsumoto, T. Otani, Y. Toyama and Y. Suda, "Knee flexion contracture will lead to mechanical overload in both limbs: A simulation study using gait analysis", The Knee, vol. 15, (2008), pp. 467-472

[14] J. Hashemi, R. Breighner, N. Chandrashekar, D. M. Hardy, A. M. Chaudhari, S. J. Shultz, J. R. Slauterbeckf and B. D. Beynnon, "Hip extension, knee flexion paradox: A new mechanism for noncontact ACL injury", Journal of Biomechanics, vol. 44, (2011), pp. 577-585

[15] H. J. Hermens, B. Freriks, C. Disselhorst-Klug and G. Rau, "Development of recommendations for SEMG sensors and sensor placement procedures", Journal of Electromyography and Kinesiology, vol. 10, (2000), pp. 361-374

[16] K. R. Kaufman, K. An, W. J. Litchy, B. F. Morrey and E. Y. S. Chao, "Dynamic joint forces during knee isokinetic exercise", The American Journal of Sport Medicine, vol. 19, no. 3, (1991).

[17] P. Konrad and The ABC of EMG, "A Practical Introduction to Kinesiological Electromyography", Version 1.0, (2005).

[18] A. D. Kuo, "A least-squares estimation approach to improving the precision of inverse dynamics computations", Journal Biomech Eng, vol. 120, no. 1, (1998), pp. 148-59

[19] A. Leardini, A. Cappozzo, F. Catani, S. Toksvig-Larsen, A. Petitto and V. Sforza, "Validation of a functional method for the estimation of hip joint center location", Journal Biomech, vol. 32, no. 1, (1999), pp. 99-103

[20] G. Mc Ginty, J. J. Irrgang and D. Pezzullo, "Biomechanical considerations for rehabilitation of the knee", Clinical Biomechanics, vol. 15, (2000), pp. 160-166

[21] E. G. Meyer and R. C. Haut R. C, "Anterior cruciate ligament injury induced by internal tibial torsion or tibiofemoral compression”, Journal of Biomechanics, vol. 41, (2008), pp. 3377-3383

[22] J. P. Paul, "Approaches to design: force actions transmitted by joints in the human body", Proceedings of the Royal Society of London, vol. 192, (1976), pp. 163-172

[23] J. G. Richards, "The measurement of human motion: a comparison of commercially available systems", Hum Movement Sci, vol. 18, no. 5, (1999), pp. 589-602.

[24] R. Riemer, T. Elizabeth, Hsiao-Wecksler and X. Zhang, "Uncertainties in inverse dynamics solutions: A comprehensive analysis and an application to gait", Gait \& Posture, vol. 27, (2008), pp. 578-588

[25] D. E. Rosenthal and M. A. Sherman, "High performance multibody simulations via symbolic equation manipulation and Kane's method", The Journal of the Astronautical Sciences, vol. 34, no. 3, (1986), pp. 223-239.

[26] S. J. G. Taylor and P. S. Walker, "Forces and moments telemetered from two distal femoral replacements during various activities", Journal of Biomechanics, (2001), pp. 839-848.

[27] A. D. Wibawa, N. Verdonschot, J. P. K. Halbertsma, R. L. Diercks and G. J. Verkerke, "Lower Limb Musculoskeletal Model Validation during One Legged Forward Hopping and Side Jumping in Healthy Subjects using EMG”, HCI International of the series Communications in Computer and Information Science, vol. 373, (2013), pp 557-560.

[28] L. H. Ting, S. A. Chvatal, S. A. Safavynia and J. L. McKay, "Review and perspective: neuromechanical considerations for predicting muscle activation patterns for movement., International Journa Numer. Meth. Biomed. Engng, (2012). 
International Journal of Bio-Science and Bio-Technology Vol.8, No.6 (2016) 